\title{
Factores pronósticos del resultado clínico de las prótesis de cabeza de radio
}

\author{
DOI: http:dx.doi.org/10.37315/SOTOCAV201928054125 \\ J. SEVIL DE LA TORRE, L. AGUILELLA FERNÁNDEZ, M. SOLER PEIRÓ. \\ HOSPITAL UNIVERSITARIO DE LA RIBERA, SERVICIO DE CIRUGÍA ORTOPÉDICA Y TRAUMATOLOGÍA. ALZIRA (VALENCIA).
}

\begin{abstract}
Resumen.
Objetivo. El sobredimensionamiento de las prótesis de cabeza del radio se ha asociado a resultados clínicos no satisfactorios junto a erosiones de capitellum. El objetivo del estudio es identificar los factores, tanto clínicos como radiológicos que han influido en nuestra serie.
\end{abstract}

Material y método. Estudio analítico transversal de una muestra de 31 pacientes entre 2005 y 2017 con un seguimiento medio de 5 años. Análisis de variables cualititativas de edad, sexo, tipo de lesión y tipo de tratamiento utilizado y análisis del tamaño del implante, mediante mediciones radiológicas (implante y margen proximal de la fosa sigmoidea cubital y ángulo ulnohumeral lateral) realizadas por evaluadores independientes, con los resultados obtenidos en escalas de valoracion MEPS y DASH y rangos de movilidad explorados al paciente.

Resultados. El tipo de fractura puede condicionar la funcionalidad clínica sin obtener diferencias estadísticamente significativas entre ellas $(p>0,05)$. La altura del implante estuvo alineada con el margen proximal en 16 casos, superior en 12 (media de $3 \mathrm{~mm}$, DS 1,5) e inferior en 3 (media de $3,4 \mathrm{~mm}$, DS de 1,7). El ángulo medio ulnohumeral lateral se obtuvo en 12 casos un aumento medio de $2,2^{\circ}$ con una disminución de flexión y supinacion asociada estadísticamente significativa $(p<0,05)$.

Conclusiones. Un alargamiento o acortamiento que no supere una media de $3 \mathrm{~mm}$ no ha demostrado alterar los resultados clínicos en nuestra serie, mientras que una apertura ulno-humeral lateral repercute negativamente sobre la flexión y la supinación.

Palabras clave: Prótesis cabeza radio, fractura cabeza radio

\section{Summary.}

Introduction. Overlengthening of a radial head implant has been associated with less than optimal clinical outcomes and capitellar erosions. The purpose of this study was to identify which factors, both clinical and radiological, have influenced the clinical results in our series.

Material and methods. Analytical study of a sample of 31 patients between 2005 and 2017 with an average follow-up of 5 years. Analysis of qualitative variables of age, sex, type of lesion and type of treatment used and analysis of the lenght of the implant by means of radiological measurements (implant and proximal margin of the lesser sigmoid notch/ lateral ulnohumeral angle) by independent evaluators with the updated results on scales of MEPS and DASH assessment and mobility ranges explored to the patient.

Results. No significant differences depending on the type of lesion nor on the type of treatment were found. Implant length was aligned with the proximal margin of the lesser sigmoid notch in 16 cases, was higher in 12 (mean 3 mm, SD 1.5) and inferior in 3 (mean $3.4 \mathrm{~mm}$, SD 1.7). The lateral ulnohumeral joint opening angle was increased an average range of $2,2^{\circ}$ and this has shown to have a significant correlation with a reduction in the range of mobility for flexion and supination $(p<0,05)$.

Conclusions. An overlengthening or shortening up to $3 \mathrm{~mm}$ has not shown to alter the clinical results in our series, while an excessive lateral ulnohumeral joint opening has a negative effect on flexion and supination mobility.

Correspondencia:

Jorge Sevil de la Torre

Departamento de Cirugía Ortopédica y Traumatología Hospital

Universitario de la Ribera.

Ctra. Corbera km 1. 46600 Alzira, Valencia. España.

Email: jorgesevildelatorre@gmail.com 


\section{Introducción}

En la actualidad el tratamiento de las fracturas de cabeza de radio se basa fundamentalmente en si es sintetizable la fractura y en la estabilidad del codo, por lo tanto, para su correcta indicación es necesario el conocimiento de la anatomía y la biomecánica para entender la función de la cabeza del radio en la estabilidad del codo y el antebrazo.

La fuerza longitudinal se transmite desde la muñeca al codo con un reparto entre el radio, cúbito y el ligamento interóseo (estabilizador principal del antebrazo), sobre todo en los casos de resección de la cabeza del radio, evitando la migración proximal del mismo que provocaría síntomas en la articulación radiocubital distal. ${ }^{1-2}$

Las fracturas de cabeza de radio suponen el $20-30 \%$ del total de las fracturas de codo, siendo el $3 \%$ del total de las fracturas. A su vez el $30 \%$ se asocian a lesiones que repercuten en la estabilidad del codo como por ejemplo: lesiones del carpo y $\mathrm{ARCD}$ (articulación radio-cubital distal), lesiones de la membrana interósea (Essex-Lopestri), fractura-luxación de Monteggia y lesiones de ligamentos colaterales medial y lateral del codo. ${ }^{2}$

En este último porcentaje de fracturas es donde radica la importancia de conservarla, ya sea mediante la reducción abierta y osteosíntesis o bien mediante artroplastia, dependiendo de si existe o no posibilidad de reconstrucción según las características de la fractura.

Para ello, Masson elaboró una clasificación basándose en hallazgos radiológicos, que más tarde se modificó atendiendo al patrón de fractura intraoperatorio y los hallazgos de lesiones asociadas.

Sin embargo, existen otros factores, a parte de los anteriores, que condicionan la toma de decisiones de nuestro tratamiento. Conocer los requerimientos funcionales que el paciente va a exigir tras la intervención es uno de los pilares fundamentales para conseguir un grado de satisfacción óptimo. Debemos individualizar entre un paciente joven, con exigencias deportivas y laborales altas; y un paciente anciano con una actividad diaria que normalmente requiera someter al codo a movimiento $y$ actividades menos exigentes.

La elección de la artroplastia de cabeza de radio en los casos indicados conlleva, a su vez, la toma de decisiones a cerca del material y de su correcta implantación.

Estudios recientes han comprobado que los implantes metálicos presentan mejores resultados a largo plazo, en comparación con los implantes de silicona, proporcionando mayor estabilidad y menos aflojamiento y deformidad del implante. Dentro de los implantes metálicos, los bipoles presentar mayores ventajas tanto en la adaptación como en la congruencia articular comparados con los monobloque. ${ }^{1-3}$

En cuanto a la colocación de la prótesis se ha observado que el sobredimensionamiento se relaciona con dolor, pérdida de flexión y signos degenerativos ulnohumerales (Fig. 1), pero no hay artículos ni protocolos descritos que especifiquen cuáles son las medidas óptimas para la implantación de la prótesis o a partir de que altura el sobredimensionamiento no es tolerable y como influye todo ello en el paciente. 3-4-5-6-7.

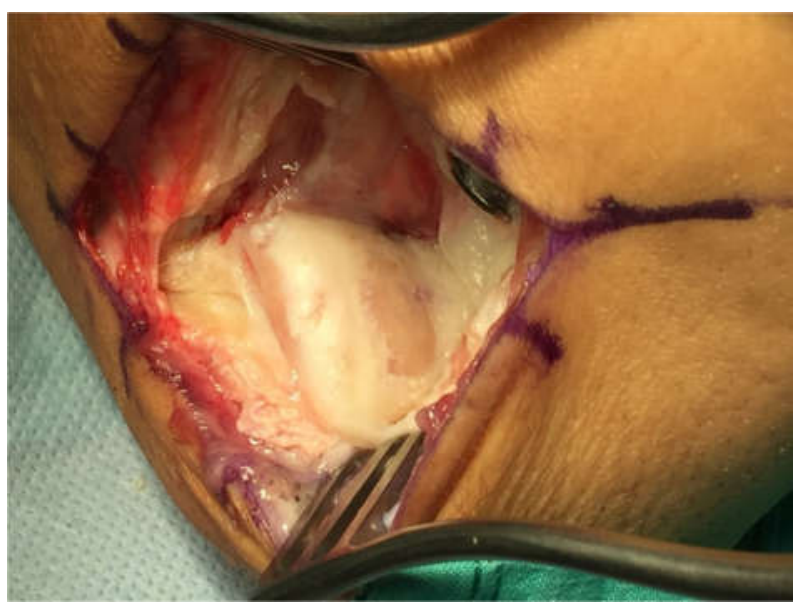

Figura 1. Erosión en el capitellum en una cirugía de revisión por overstuffing de prótesis de cabeza de radio.

El objetivo de nuestro estudio es analizar si existe relación del infra o sobredimensionamiento de las prótesis de cabeza de radio con la funcionalidad postoperatoria del codo intervenido (Fig 2A-2B), identificando si hay factores clínicos y radiológicos que influyen en nuestra serie de casos.

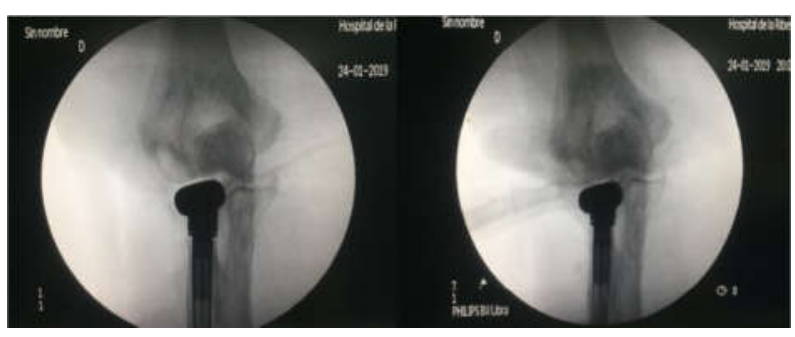

Figura 2A. Escopia intraoperatoria donde se observa el overstuffing de la prótesis de cabeza de radio. 2B. Escopia intraoperatoria con su correcta implantación

\section{Material y Métodos}

Se realizó un estudio analítico de carácter transversal durante un período de 2 meses en los que se llevó a cabo la recogida de datos de nuestros pacientes.

Se redactó un consentimiento informado que fue aceptado por el Comité de Docencia de nuestro Centro $\mathrm{y}$ que fue firmado $\mathrm{y}$ aceptado por los pacientes que accedieron al estudio.

Durante el período 2005-2017 se colocaron 49 implantes de cabeza de radio (MoPyC, Bioprofile, Tornier) en pacientes del Departamento 11 del Área de Salud de la provincia de Valencia. Los criterios de inclusión fueron aquellos pacientes intervenidos de prótesis de cabeza de radio que pertenecieran a nuestra área. Como criterio de exclusión únicamente se 
consideró a aquellos pacientes con imposibilidad para realizar el estudio (pacientes de otra área, domicilio en el extranjero, fallecimiento). Accedieron a ser evaluados 31 pacientes, 21 mujeres y 10 varones, con una edad media de 53 años (rango 17-78 años) y un seguimiento medio de 5 años.

Se recogieron por un lado variables cualitativas de cada paciente: sexo, edad, tipo de fractura, indicación seleccionada para el tratamiento.

Para la medición de las dimensiones de la cabeza de radio de nuestros implantes se sometió a los pacientes a un estudio radiológico, tanto del codo intervenido como del codo contralateral, a $45^{\circ}$ de flexión y pronosupinación neutra en ambos codos, de esta forma conseguíamos una posición estándar para que pudiera ser realizada por todos nuestros pacientes.9 Las mediciones fueron realizadas por dos cirujanos pertenecientes al servicio de COT de nuestro Centro, llevándose a cabo una relación interobservador y posteriormente obteniendo el valor medio de cada medición para llevar a cabo el estudio.

El objetivo del estudio radiologico era comprobar si existía un aumento del espacio ulnohumeral con distintas medidas:

\section{- $\quad$ Angulación ulnohumeral lateral4-5-7-8 (Fig 3).}

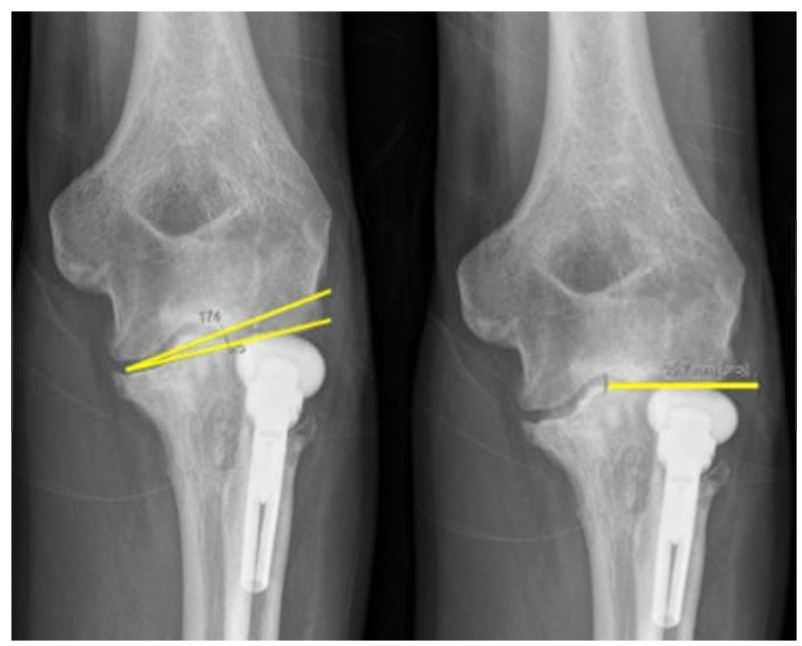

Figura 3 y 4. Medición radiográfica ángulo ulnohumeral lateral. Y medicion de la distancia del implante con el borde proximal de la fosa sigmoidea cubital.

- $\quad$ Altura del implante con respecto al margen proximal de la fosa sigmoidea6 (Fig 4).

Todas estas medidas se realizaron tanto en el codo intervenido como en el contralateral sano, el cuál fue tomado como referencia anatómica. Se recogieron los datos absolutos y la diferencia entre cada uno de ellos y se consideró para el análisis el valor medio de las mediciones.
Para analizar la funcionalidad postquirúrgica se tuvo en cuenta la lateralidad de la lesión y si había sufrido anteriormente una lesión de codo. Como variable cuantitativa objetiva se midió en grados el rango de movilidad mediante una exploración al paciente. Como variable cuantitativa subjetiva se utilizaron las siguientes escalas de valoración: Mayo Elbow Performance Score (MEPS), DASH.

\section{Análisis estadístico}

El análisis estadístico fue llevado a cabo mediante el progama IBM SPSS Windows, versión 22 (IBM Corp, Armonk, N.Y., USA).

Como estadístico de referencia para los valores cuantitativos descriptivos se utilizó la media aritmética de los datos obtenidos con unos intervalos de confianza del $95 \%$. Los valores cualitativos se expresaron como frecuencias.Para analizar el nivel de concordancia interobservador en las mediciones radiológicas realizadas se utilizó el coeficiente de Kappa.Como estadístico de prueba para variables no paramétricas se utilizó la prueba de Kruskall Wallis con un nivel de significación de 0,05.

\section{Resultados}

Dentro de la muestra de 31 pacientes, atendiendo al tipo de lesión se obtuvieron 5 casos Masson tipo III, 6 casos Masson tipo IV, 12 casos de tríada de codo y 8 casos con fractura de cabeza de radio asociada a fractura de cúbito proximal. Si nos basamos en el tipo de tratamiento utilizado, en 9 casos únicamente se sustituyó la cabeza de radio, en 12 casos se asoció a la prótesis de cabeza de radio la osteosíntesis de coronoides y la reparación ligamentosa, y en 10 casos asoció a una osteosíntesis de cúbito por fractura asociada.

A la hora de comparar el tipo de fractura con los resultados clínicos de los pacientes se obtuvieron buenos resultados, de acuerdo con las escalas de valoración, siendo un DASH medio de $23(15-31)$ IC 95\% y un MEPS medio de 83 (76-89) IC 95\% sin obtener diferencias estadísticamente significativas entre los tipos de fractura, $p>0,05$ en ambas escalas, (Tabla IA-1B), aunque se obtuvieron mejores resultados en aquellas fracturas sin lesiones ligamentosas ni otras fracturas

asociadas. 

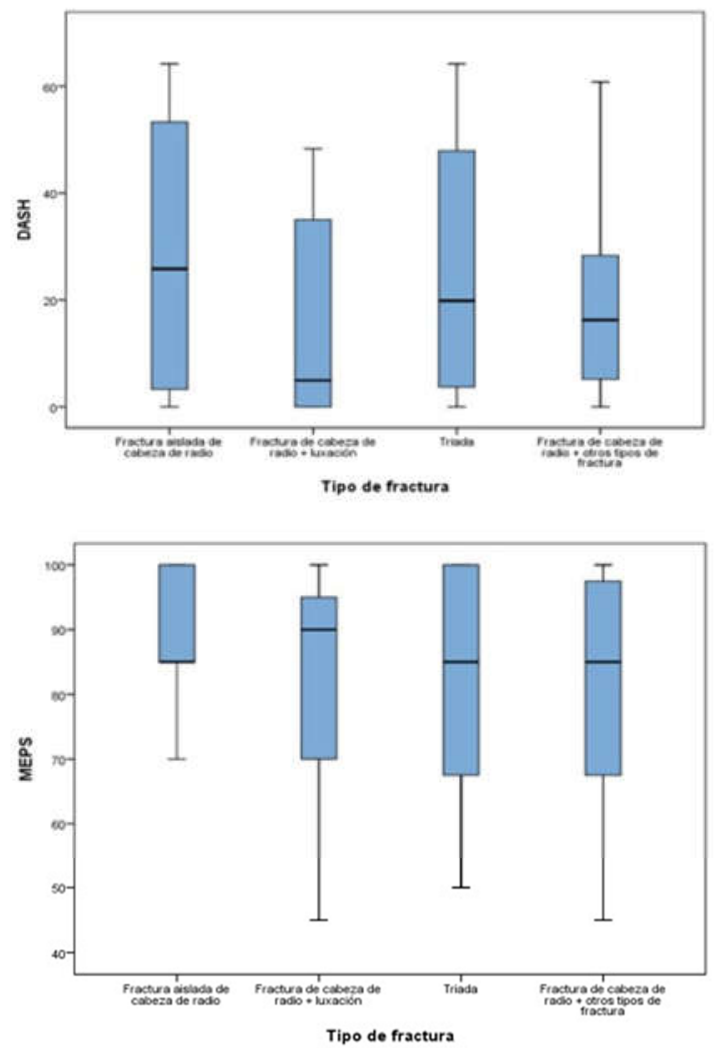

Tabla IA. Gráfica de estudio de relación entre escala DASH y tipo de fractura.

Tabla IB. Gráfica de estudio de relación entre escala MEPS y tipo de fractura.

Con respecto a los rangos de movilidad obtenidos, fueron satisfactorios, una flexión media de $125^{\circ}(119-$ 130) IC $95 \%$, una extensión media de $-13^{\circ}(-8 /-18)$ IC $95 \%$, una pronación medio de $86^{\circ}(83-89)$ IC $95 \%$ y una supinación media de $80^{\circ}$ (73-86) IC 95\%, sin embargo, igual que ocurría con las escalas de valoración no se obtuvieron diferencias significativamente estadísticas entre los tipos de fractura, $p>0,05$, aunque también se obtuvieron mejores resultados en aquellas fracturas menos complejas (Tablas IIA-2B-2C-2D).

Para llevar a cabo el estudio de las dimensiones de la cabeza de radio fueron realizadas mediciones radiológicas en el codo intervenido y en el codo contralateral mediantes 2 observadores independientes para los que se calculó el índice Kappa para valorar el nivel de concordancia. Los resultados obtenidos,
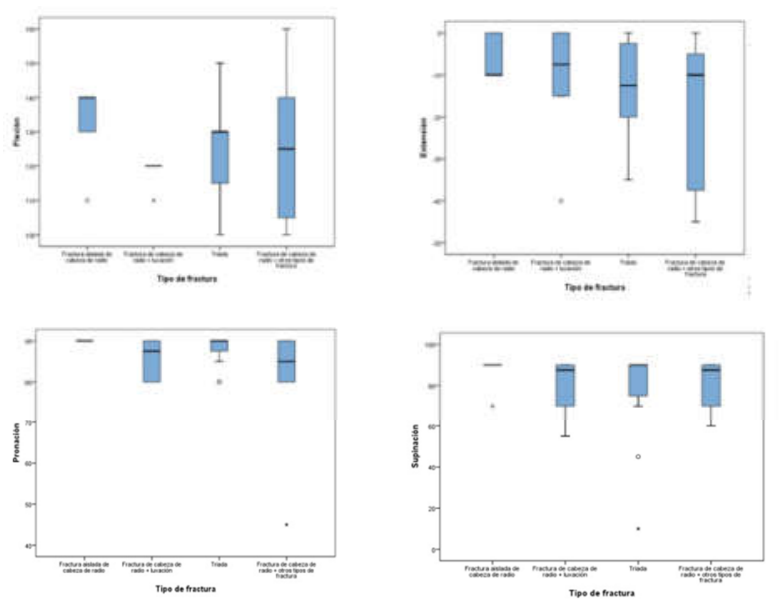

Tabla IIA. Gráfica de estudio de relación de flexión y tipo de fractura.

Tabla IIB. Gráfica de estudio de relación de extensión y tipo de fractura.

Tabla IIC. Gráfica de estudio de relación de pronación y tipo de fractura.

Tabla IID. Gráfica de estudio de relación de supinación y tipo de fractura.

midiendo la distancia entre el borde proximal de la fosa sigmoidea cubital y el implante, fueron superior en 12 casos (media de $3 \mathrm{~mm}$, DS 1,5), inferior en 3 casos (medio de $3,4 \mathrm{~mm}$, DS 1,7 ) y alineado con el borde proximal en 16 casos, sin obtener diferencias estadísticamente significativas con respecto a los rangos de movilidad y escalas clínicas, siempre que el alargamiento o acortamiento no sobrepasara los $3 \mathrm{~mm}$ (Tabla III).

Si atendemos a la apertura del ángulo ulnohumeral lateral el ángulo medio en los codos intervenidos fue de $10,7^{\circ}$ (DS 2,6) siendo de $12,2^{\circ}$ en los codos contralaterales no intervenidos. En 12 pacientes el ángulo medio era de $14,2^{\circ}$, por lo tanto, un ángulo medio superior de $2^{\circ}$ con respecto al codo contralateral, obteniendo diferencias estadísticamente significativas $(p<0,05)$ en el empeoramiento de la flexión y la supinación en $7^{\circ}$ y $12^{\circ}$ respectivamente. Sin embargo, no se obtuvieron diferencias significativamente estadísticas con respecto a las escalas de valoración clínica $(p>0,05)$ (Tabla IV). 


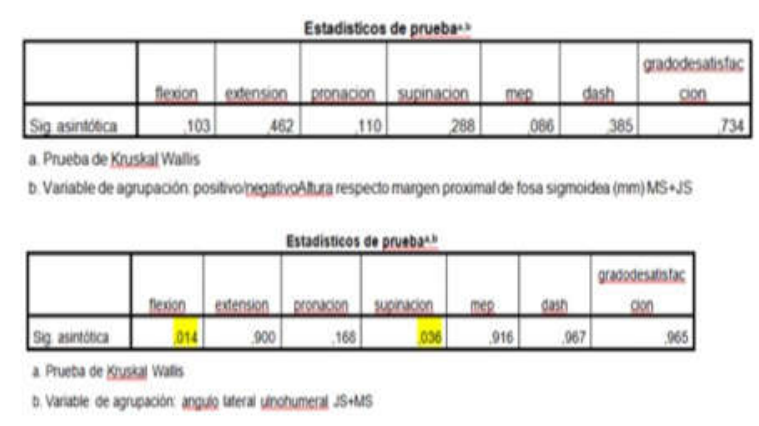

Tabla III. Resultado análisis estadístico de distancia del implante con el borde proximal de la fosa sigmoidea.

Tabla IV. Resultado análisis estadístico de ángulo ulnohumeral lateral.

\section{Discusión}

El objetivo del cirujano ortopédico en este tipo de fracturas de codo es restituir la funcionalidad de codo lo más similar posible a la que tenía el paciente antes de sufrir el traumatismo. Para ello, en aquellos casos de fractura de cabeza de radio no sintetizable asociado a su vez a inestabilidad de codo, la sustitución de cabeza de radio es indispensable para mantener la correcta longitud del radio y conservar una correcta cinématica y presión intraarticular evitando así una enfermedad degenerativa precoz como resultados del estrés del cartílago.

A través de nuestro trabajo hemos analizado, mediante un estudio radiológico comparativo junto con los resultados de las escalas de valoración, cómo una incorrecta longitud del implante, puede acarrear una serie de complicaciones como puede ser la inestabilidad en valgo residual que se produce con el acortamiento del radio; o la rigidez articular, varo residual y dolor junto a un proceso degenerativo precoz que ocurriría con un sobredimensionamiento de la prótesis de cabeza de radio. ${ }^{1-3-10-11}$

Es evidente que aunque en nuestra serie no hayamos obtenido resultados estadísticamente significativos entre el tipo de fractura y los resultados funcionales se ha observado unos mejores resultados en aquellas sustituciones de cabeza de radio con patrones de fractura menos complejos que en aquellos asociados a inestabilidades u otro tipo de fracturas de codo, por lo tanto, es discutible que en los resultados obtenidos en las mediciones de cabeza de radio hayan influido las lesiones asociadas y el alcance del traumatismo inicial. Otro factor a analizar sería si ha influido que la muestra no haya sido realizada en su totalidad por el mismo cirujano, siendo, sin embargo, una muestra bastante homogénea.

En nuestra serie hemos obtenido que un alargamiento o acortamiento medio que no supere los $3 \mathrm{~mm}$ no altera los resultados clínicos de nuestros pacientes, cifra que no dista de los resultados obtenidos en otras series que hablan de 2,5mm. Además, aportamos resultados estadísticamente significativos con respecto a la disminucion de la flexión y supinación con el aumento medio en nuestra serie de $2^{\circ}$ del ángulo ulnohumeral lateral. $^{7-8}$

Con lo que respecta al seguimiento y el grado de satisfacción de nuestros pacientes, ha sido durante un período medio de 5 años en los que se han obtenido buenos resultados, aunque en algunos de ellos el resultado radiológico no era acorde con el resultado funcional.1 Nuestro objetivo es restaurar de forma anátomica la biomecánica del codo tras la fractura, sin embargo, en ocasiones, no podemos controlar la evolución, ni las espectativas de ese paciente tras una lesión compleja de codo.

\section{Conclusiones}

El tipo de lesión influye en los resultados clínicos sin obtener diferencias estadísticamente significativas entre ellas. Un alargamiento o acortamiento que no supere los $3 \mathrm{~mm}$ de media no ha demostrado alterar los resultados clínicos en nuestra serie y, por último, el aumento de la apertura ulnohumeral lateral con respecto al codo contralateral disminuye el rango de flexión y supinación, sin embargo, no podemos confirmar con certeza que influya en las escalas de valoración clínica. 
SEVIL DE LA TORRE J Y COLS. Factores pronósticos del resultado clínico de las prótesis de cabeza de radio.

\section{Bibliografía}

1. Manraj Nirmal Kaur, Joy C MacDermid, Ruby R Grewal, Paul W Stratford, and Linda J Woodhouse. Functional outcomes post-radial head arthroplasty: a systematic review of literature. Shoulder Elbow 2014; 6(2): $108-18$.

2. Kimberly M, Robert N H. Fracturas y luxaciones de codo. Rockwood and Green's fractures in adults, 20075 ed. Vol1: 921-53.

3. Van Glabbeek F, Van Riet RP, Baumfeld JA, Neale PG, O'Driscoll SW, Morrey BF, An KN. Detrimental effects of overstuffing or understuffing with a radial head replacement in the medial collateral-ligament deficient elbow. $J$ Bone Joint Surg Am 2004; 86(12):2629-35.

4. Doornberg JN1, Linzel DS, Zurakowski D, Ring D. Reference points for radial head prosthesis size. J Hand Surg Am 2006; 31(1):53-7.

5. Rowland AS1, Athwal GS, MacDermid JC, King GJ. Lateral ulnohumeral joint space widening is not diagnostic of radial head arthroplasty overstuffing. Hand Surg Am 2007; 32(5):637-41.

6. van Riet RP, van Glabbeek F, de Weerdt W, Oemar J, Bortier H. Validation of the lesser sigmoid notch of the ulna as a reference point for accurate placement of a prosthesis for the head of the radius: a cadaver study. $J$ Bone Joint Surg Br 2007 Mar; 89(3):413-6.

7. Frank SG, Grewal R, Johnson J, Faber KJ, King GJ, Athwal GS. Determination of correct implant size in radial head arthroplasty to avoid overlengthening. J Bone Joint Surg Am 2009 Jul; 91(7):1738-46.

8. Athwal, George S., MD; Frank, Simon G; Grewal, Ruby, MD; Faber, Kenneth J., MD; Johnson, James, Ph; King, Graham J.W. Determination of correct implant size in radial head arthroplasty to avoid overlengthening. Surgical technique. JBJS 2010; 92 Supp 1 (2):250-7.

9. Athwal GS, Rouleau DM, MacDermid JC, King GJ. Contralateral elbow radiographs can reliably diagnose radial head implant overlengthening. J Bone Joint Surg Am 2011; 93(14):1339-46.

10. Abdulla I, Langohr GD, Gladwell M, Yeung C, Faber KJ, King GJ, Athwal GS. The effect of fracture comminution on the reliability and accuracy of radial head sizing. J Shoulder Elbow Surg 2015; 24(3):364-8.

11. Kim HM, Roush EP, Kiser C. Intraoperative fluoroscopic assessment of proper prosthetic radial head height. $J$ Shoulder Elbow Surg 2016; 25(11):1874-81. 